\title{
DETERMINACIÓN DE LA CONFIABILIDAD EN UNA PRUEBA PARA LA MEDICIÓN DE DOLOR EN ADULTAS MAYORES
}

\author{
Luis Carlos Solano Mora, Olman Vargas Zumbado, y Nórbel Román Garita \\ Universidad de Costa Rica, San José, Costa Rica \\ E-mail:lcsolano@cariari.ucr.ac.cr
}

\begin{abstract}
Resumen
Solano Mora, L. C., Vargas Zumbado, O., y Román Garita, N. (2001). Determinación de la confiabilidad de una prueba para la medición del dolor en adultas mayores. Revista de Ciencias del Ejercicio y la Salud, 1(1), 42-49. Se realizó un estudio para determinar la confiabilidad de una prueba para medir dolor en adultas mayores. Se utilizó un grupo de 12 adultas mayores $(N=12)$, con una edad promedio de 63.58 años (D.S. \pm 10.08$)$. Se realizó un pretest para observar el nivel de dolor inicial de las sujetos en las rodillas, el cual consistió en ejecutar cuatro movimientos (poniéndose de pie, caminando, subiendo gradas y bajando gradas). Después de realizar un solo movimiento, la ejecutante indicaba cuánto dolor sentía entre 0 (sin dolor) y 10 (dolor severo); posteriormente, después de estar en reposo durante dos horas, se les aplicó el post test, en donde se repitió todo el proceso anterior. Para observar si existieron diferencias estadísticamente significativas entre los promedios de ambas mediciones, fue aplicada una ANOVA de una vía de medidas repetidas y, para obtener la confiabilidad de la prueba, se aplicó el coeficiente $\boldsymbol{r}$ de Pearson. Los resultados no mostraron diferencias estadísticamente significativas entre los promedios de ambas mediciones para 11 g.l. y una $p<.001$. La prueba $\boldsymbol{r}$ de Pearson mostró que los coeficientes obtenidos para los cuatro movimientos (poniéndose de pie $=.73$, caminando $=.77$, subiendo gradas $=.92$ y bajando gradas $=.75)$ fueron mayores que el valor crítico de la Tabla de Pearson $(11$ g.l., $\alpha=.01$, valor crítico $=$ .634). Se concluye que el dolor es medible bajo condiciones controladas y que la prueba de dolor es realmente confiable para cada uno de los cuatro movimientos ejecutados. PALABRAS CLAVES: confiabilidad, prueba de dolor.
\end{abstract}

\section{INTRODUCCION}

Algunas áreas dentro del campo de la medicina, son reconocidas especialmente por su dificultad para ser observadas así como por su dificultad a la hora de ser medidas o cuantificadas.

Específicamente, un área que ha provocado contradicciones y problemas en las ciencias médicas a la hora de ser medida, es el área del dolor.

Antes de dar una explicación al problema de la medición del dolor, es importante realizar primero que nada, una descripción de la definición del término dolor y sus medios de transmisión.

El Sistema Nervioso Central busca obtener información tanto del medio interno como del medio externo para activar los diferentes sistemas del cuerpo y así, mantener la homeostasis del organismo. A los modos o procesos por los cuales se obtiene dicha información se les llama mecanismos de captura sensoriales (Aguilar, 1991). Uno de estos procesos de obtención de información es la somestesia o sensibilidad somática, la cual se conceptualiza "como el conjunto de sensaciones producidas por la estimulación de receptores localizados en la piel" ; esta se subdivide en mecanorrecepción o sensibilidad táctil, termorrecepción o sensibilidad térmica y nocicepción o sensibilidad al dolor (Aguilar, 1991). Este estudio se abocará a este último punto; ahora bien, $i$ cómo se define el término dolor ?

La definición conceptual del dolor aceptada internacionalmente, indica que el 
"dolor es una sensación y una experiencia emocional asociada con amenaza o daño real a los tejidos" (Zwetnow, 1980). Por su parte, Prieto (1991, pp. 166-167) manifiesta una definición muy similar a la anterior, la cual indica que "el dolor es una sensación originada por algún estímulo que amenaza con lesionar o lesiona (estímulo nociceptivo) algún tejido. Es una experiencia que se acompaña de marcada molestia, siendo esta característica lo que la diferencia de otras modalidades sensoriales".

Cabe agregar que el autor Zwetnow (1980), indicó que hay diferentes aspectos que influyen en la percepción del dolor entre los individuos, en donde se destacan:

1. La personalidad.

2. Experiencias anteriores.

3. El momento donde se percibió el dolor.

4. Las diferentes reacciones de las personas ante el dolor.

5. El nivel cultural y social del sujeto.

Esto último es soportado por Prieto (1991, p. 167), quien reconoce que la manera como se responde o reacciona ante el dolor es muy diferente entre los organismos y va a depender entre otras cosas de "la intensidad, la frecuencia, la duración y la naturaleza del estímulo", además de otros aspectos que podrían influir en la percepción del dolor, como son "la raza, la edad, el sexo, la personalidad, el estado afectivo, la atención y los valores culturales de evaluación del dolor".

Es importante acotar que así como hay diferencias en la percepción del dolor, también hay diferencias en cuanto a las fibras que transmiten los impulsos aferentes del mismo, lo que en última instancia va a definir el tipo de dolor percibido por una persona.

En el tema del dolor hay una serie de estudios experimentales clásicos como los de Gasser y Erlanger (1929); Zotterman (1933) y Heinbecker et al. (1934), los cuales establecieron que el dolor es transmitido hacia el sistema nervioso central por medio de dos sistemas de fibras aferentes, específicamente las fibras delta y las fibras C.

Estos dos tipos de fibras trasmiten formas de dolor muy diferentes, tal y como se observa en otro estudio clásico realizado por Collins et al. (1960) en pacientes a los que se les tenía que aplicar una cordotomía. Anterior a la cordotomía, a los pacientes se les disecó el ciático poplíteo interno en el transcurso de la anestesia local, estimulándolo después con un par de electrodos, en donde los pacientes recibían diferentes tipos de impulsos o estímulos eléctricos, unos aislados y otros repetitivos. El estudio demostró que a estímulos bajos los pacientes percibían una vibración leve; a estímulos más elevados producía una sensación urente o quemante en donde se activaron las fibras delta y, según el reporte, fue tolerado en su mayoría por los pacientes. Caso contrario ocurrió cuando se dio una estimulación aun más alta, donde se activaron las fibras C. En la sección del experimento en que se les sometía a impulsos aislados, estos les provocaron dolores muy intensos; pero cuando se pasó a los estímulos iterativos, estos fueron tan intensos y dolorosos, que los pacientes desistían de seguir con el experimento, pudiéndose apreciar que este estudio logra separar el tipo de dolor que es agudo pero normalmente tolerable de las fibras delta y el dolor totalmente intolerable y lacerante de las fibras C.

Con respecto a la transmisión de señales nerviosas de dolor, (Zwetnow, 1980, p. 113) manifestó que hace un tiempo, "las astas posteriores eran consideradas como estaciones de relevo para el influjo aferente de señales solamente", hasta que unos estudios experimentales en animales, mostraron que "las astas posteriores son la estación de relevo sináptico más importante en todo el sistema de transmisión del dolor".

Sin embargo, Verbiest (1980, p. 6061) ha indicado que "las experiencias ante el dolor y las respuestas al mismo debido a alteración mecánica aguda son más 
uniformes que en circunstancias de dolor más crónico".

Además, Zwetnow (1980) mencionó que aunque se hayan hecho importantes avances en el área del dolor, su medición siempre ha presentado un gran problema.

Este último punto es clave para la presente investigación, pues sugiere que la evidencia disponible no es precisa ni suficiente para poder evaluar el dolor.

Acorde con lo anterior, Schürmann (1980, p. 41) indicó que aun cuando se ha observado en la fisiología que el dolor es medible, no se cuenta actualmente con métodos lo suficientemente precisos para mostrar "en forma objetiva un estado crónico de dolor y de sufrimiento, ni tampoco hay técnicas exactas para medir la intensidad del dolor que siente el paciente".

Pero entonces, $i$ qué características posee el dolor qué es tan difícil de cuantificar ? Una explicación de esto nos la brinda Zwetnow (1980, p. 121), quien manifiesta que "el dolor es una de las pocas percepciones que pueden provenir del interior del cuerpo y que carece de un estímulo específico, es decir, puede ser producido en muchas formas diferentes".

Además, el mismo Zwetnow (1980, p. 131) es enfático en reconocer que "la medición del dolor se caracteriza por dificultades conceptuales y metodológicas. No obstante, es vital para el diagnóstico, tratamiento y entendimiento teórico"; asimismo, indica que "el número de métodos actuales pone de manifiesto que ningún sistema es bueno" y que por lo tanto, cada investigador necesita poseer diferentes métodos para evaluar distintos aspectos del dolor según sea el caso.

Este último punto es reforzado por Chapman y Syrjala (1990, p. 580) quienes indicaron que hay personas que por sus características, necesitan de un tipo particular de tratamiento más que de otro, lo que podría implicar que necesitan también de un tipo de medición específica de acuerdo con el estímulo o la condición dolorosa que presenten, de ahí que los autores concluyan que, "no hay una medición estándar para cuantificar el dolor en todas las circunstancias". Cabe agregar que esto último también fue afirmado por O'Connor y Cook (1999).

Entonces, ¿ qué importancia tiene el obtener el criterio estadístico de la confiabilidad en una prueba de dolor ? Antes de exponer este punto, es necesario explicar qué significa el término confiabilidad para así observar cuál es su relevancia en esta investigación.

Según Ary et al. (1989, pp. 214,216), "la confiabilidad de un instrumento de medición es el grado de uniformidad con que cumple su cometido", o lo que es lo mismo, "denota el grado de congruencia con que se realiza una medición".

Otros autores como Hernández et al. (1991, p. 242), de manera similar a los mencionados anteriormente, establecen que "la confiabilidad de un instrumento de medición se refiere al grado en que su aplicación repetida al mismo sujeto u objeto, produce iguales resultados".

Por otra parte, Thomas y Nelson, (1985, p. 349) también han indicado que la confiabilidad es el grado de consistencia de un instrumento de medición.

De igual forma, Kerlinger (1988) ha manifiestado que los términos seguridad, consistencia, predictibilidad y exactitud están incluidos en la definición del término confiabilidad.

Asimismo, Ary et al. (1989) agregan que la confiabilidad puede verse afectada por los errores de tipo aleatorio, los cuales ocurren casualmente y podrían variar los puntajes que obtengan cualesquiera de los sujetos de manera impredecible; destacándose así, tres tipos de errores que provocan alteraciones en los datos obtenidos:

1. Variación del sujeto entre una y otra medición.

2. Alteración del protocolo de la prueba entre las mediciones.

3. Puntuaciones muy variadas producto de muestras muy pequeñas de sujetos 
Entonces, de acuerdo con Ary et al. (1989) "la confiabilidad se ocupa del efecto de tales errores aleatorios sobre la congruencia de las puntuaciones".

Kerlinger (1988, p. 470), indica que se puede instaurar la definición de confiabilidad bajo tres puntos. Un primer punto sugiere una definición del término confiabilidad como la estabilidad, seguridad y predictibilidad de una prueba; un segundo punto va enfocado en la confiabilidad como una medida de exactitud, es decir, si los puntajes obtenidos son los puntajes verdaderos del objeto que se estaba midiendo y un tercer punto que define la confiabilidad "como la ausencia relativa de errores de medición en un instrumento"; en resumen, "la confiabilidad es la exactitud o precisión de un instrumento de medición".

Un detalle en extremo importante, mencionado por Ary et al. (1989, p. 216), es que a la confiabilidad "no le interesa saber si se está midiendo lo que se desea, eso es una cuestión de validez. Un instrumento de medición puede ser confiable y no obstante carecer de validez. Sin embargo, no puede ser válido si antes no es confiable". De manera similar, Thomas y Nelson, (1985) indican que una medición que no mantenga una consistencia entre sus resultados, no puede ser válida puesto que el investigador no puede confiar en los resultados.

Kerlinger (1988, p. 470) es más explícito en cuanto al valor de la confiabilidad, al indicar que "una alta confiabilidad no es garantía de buenos resultados científicos, pero no puede haber buenos resultados científicos sin confiabilidad".

Con respecto a la administración propiamente dicha de la prueba, Ary et al. $(1989$, p. 217) indicaron que se sabe "que en todas las mediciones de cualidades humanas hay cierto grado de error; entonces, los procedimientos de confiabilidad se proponen determinar hasta qué punto la incongruencia de las puntuaciones proviene del error aleatorio".
Este último concepto es igualmente mantenido por Thomas y Nelson (1985, p. 349), citando que la confiabilidad de un instrumento es analizada en "términos de su puntaje observado, su puntaje verdadero y su puntaje de error", así como por Hernández et al. (1991, p. 248), quienes citan que "es casi imposible que una medición sea perfecta; generalmente se tiene un grado de error".

De acuerdo a lo manifestado por los autores Thomas y Nelson (1985); Ary, et al.. (1989) y Hernández et al.. (1991), los datos que se obtienen en una medición son denominados puntuación observada (X), pero muy posiblemente al suministrar de nuevo la prueba, el sujeto o los sujetos podrían no obtener los mismos puntajes de su primera medición, debido muy probablemente al grado de error de la medición; ante lo cual se puede afirmar, que todos los puntajes en una medición se componen de dos factores: Uno denominado como puntuación verdadera (t) y otro denominado como puntuación de error (e), lo cual matemáticamente se expresa así:

$$
X=t+e
$$

Lord y Novick (1968, pp. 30-31), ya habían definido a la puntuación verdadera "como el valor esperado de un puntaje observado, el cual puede interpretarse como el puntaje promedio que un individuo obtendría en número infinito de mediciones independientes y repetidas".

De la misma manera, Ary, Jacobs y Razavieh (1989, p. 218) indican que la confiabilidad es teóricamente definida como "la razón de la varianza de puntuación verdadera con la de la puntuación observada en un conjunto de puntuaciones".

Con base en la información anterior, es clara la importancia de obtener el criterio estadístico de la confiabilidad en las pruebas de dolor, pues al tener los investigadores diferentes protocolos de pruebas sin contar con una valoración estadística previa, provoca que los resultados sean realmente difíciles de comparar, puesto que se están 
haciendo análisis con base en una o unas pruebas que en realidad no se sabe si son confiables, aparte que algunas veces se varía el protocolo de administración de la prueba, provocando aun más dificultad en la comparación de datos entre los investigadores. Además se ha observado en la literatura, una creciente información acerca de más pruebas para la medición del dolor; pruebas que de hecho no poseen ningún criterio estadístico, con lo cual incrementan el problema anteriormente mencionado (Chapman y Syrjala, 1990).

Ahora bien, dentro del área de interés del presente trabajo, no se encontró evidencia alguna acerca de una prueba o pruebas específicas para medir el dolor de rodillas en los adultos mayores (Revill et al., 1976; Zwetnow, 1980; Jensen et al., 1986; Chapman y Syrjala, 1990; Cook et al., 1997; O' Connor y Cook, 1999).

Este punto es importante en el tema del adulto mayor, ya que Whiting y Zernicke (1998) han observado que hay enfermedades como la osteoartrosis de rodillas, que llegan a provocar incapacidad en el adulto mayor, debido a que esta ocurre fundamentalmente en las articulaciones que sostienen el peso corporal, lo cual incide negativamente en su calidad de vida. De este modo al no poseer una prueba de dolor específica para un estímulo o problema específico, se tiene un vacío en la literatura en cuanto a que tipo de prueba pueda ser la que mejor indique el grado de evolución de una enfermedad en los adultos mayores, en este caso, la osteoartrosis de rodillas.

Por lo tanto y con base en los estudios revisados, se observa que es necesaria la creación de una prueba específica para medir el dolor de rodillas en adultos mayores y que sea confiable.

Esta investigación por consiguiente, tiene como objetivo, el obtener el nivel de confiabilidad de una prueba de dolor modificada para rodillas en cuatro diferentes movimientos, los cuales son: Poniéndose de pie; caminando; bajando gradas y subiendo gradas.
Por todo lo anterior, se establece como problema general lo siguiente:

¿ Puede una nueva prueba de dolor ser confiable para medir el dolor en adultos mayores con osteoartrosis de rodillas ?

De la misma manera, se planteó como hipótesis el siguiente enunciado:

Los puntajes de la primera medición de dolor serán consistentes con los puntajes de la segunda medición de dolor para cada uno de los cuatro movimientos medidos.

\section{Metodología}

\section{Sujetos}

Se utilizó una muestra de 12 adultas mayores sedentarias con debilidad y artrosis de rodilla de leve a muy leve y con edades entre los 51 años y los 84 años $(M=63.58 \pm$ 10.08 años).

\section{Instrumentos de medición}

Se utilizó la modalidad de un test de dolor citado por Chapman y Syrjala (1990), denominada Escala Evaluación Numérica, que es una escala que va desde cero (0), que indica que no hay presencia de dolor, hasta diez (10), que indica que el dolor percibido es extremo o intolerable.

Este test se puede aplicar para medir estados subjetivos de percepción de dolor y para efectos de esta investigación se hizo en las articulaciones de las rodillas. Está compuesto por cuatro (4) movimientos, a saber:

1. Poniéndose de pie.

2. Caminando.

3. Bajando gradas.

4. Subiendo gradas.

Para este tipo de investigación, se necesitó de un test que tuviera la capacidad de ser sensible ante los cambios que pudieran manifestar los sujetos, es decir, que fuera lo más preciso posible para observar cambios en las posiciones relativas de los sujetos 
dentro del grupo, por lo cual se optó por este tipo de escala de evaluación numérica de cero (0) a diez (10). Esta escala se aplicó para cada uno de los cuatro movimientos descritos.

\section{Procedimientos de medición}

Se escogió una muestra de 12 sujetos adultas mayores para realizar la prueba y con previo consentimiento de las mismas habiéndoles explicado en qué consistía la investigación. Una vez hecho esto, se procedió a la toma de datos iniciales (nombre y edad), para posteriormente iniciar las mediciones de las sujetos una por una, en donde se les pedía que realizaran los movimientos de manera lenta.

Finalizada la ejecución de cada movimiento, las adultas mayores observaban la Escala de Evaluación Numérica y le indicaban al investigador cuanto fue lo que sintieron de dolor entre cero (sin dolor) y diez (dolor intolerable). Posterior a este punto, las adultas mayores debían estar sentadas durante dos horas con el objetivo de que no aplicaran carga alguna sobre las articulaciones de las rodillas; cabe agregar que durante ese tiempo en que estuvieron sentadas, las señoras podían leer, escuchar música, tejer o cualquier otra actividad que implicara únicamente un movimiento mínimo; posterior a este período de espera, se les procedió a aplicar el post test de dolor en el orden en que fueron evaluadas en un inicio. Es importante aclarar, que el test se aplicó bajo la modalidad test-retest realizado el mismo día, mencionado por Thomas y Nelson (1985).

Posterior a que se recogieran todos los datos, estos fueron tabulados para realizar los procedimientos estadísticos respectivos.

\section{Análisis estadístico}

Una vez que los datos fueron tabulados, se les aplicó una ANOVA de una vía de medidas repetidas para cada movimiento, para determinar si existieron diferencias estadísticamente significativas entre los promedios del pretest y el post test; además, se aplicó una prueba $r$ de Pearson para determinar el coeficiente de confiabilidad de test-retest.

\section{RESULTADOS}

La ANOVA de una vía de medidas repetidas aplicada a cada uno de los factores observados (posiciones), mostró que no hubo diferencias estadísticamente significativas entre los promedios del pre test vs. los promedios del post test, en ninguna de las cuatro posiciones medidas. La Tabla 1 muestra los valores del pretest y del postest para cada uno de los movimientos ejecutados.

Tabla 1

Resultados de los puntajes del pretest y del postest para cada uno de los cuatro movimientos.

\begin{tabular}{lcc}
\hline & Pretest & Postest \\
\cline { 2 - 3 } Poniéndose de pie & 17 & 23 \\
Caminando & 17 & 16 \\
Subir gradas & 30 & 28 \\
Bajar gradas & 15 & 23 \\
\hline $\mathrm{p}>.001$ & &
\end{tabular}

La Tabla 2 muestra que para el movimiento poniéndose de pie, la Fc fue de 0.79; para caminar la Fc fue de 0.04; para subir gradas la Fc fue de 0.22 y para bajar gradas la Fc fue de 2.05, todas estas fueron significativamente menores que la Ft, la cual tuvo un valor de 19.7 para 11 g.l. y una $\mathrm{p}>$ de .001 (Ver Tabla 2).

Tabla 2

Análisis de Varianza Obtenidos en cada Factor.

\begin{tabular}{lcc}
\hline & Fc & Ft (1,11) \\
\cline { 2 - 3 } Poniéndose de pie & 0.79 & 19.7 \\
Caminando & 0.04 & 19.7 \\
Subiendo Gradas & 0.22 & 19.7 \\
Bajando Gradas & 2.05 & 19.7 \\
\hline $\mathrm{p}>.001$ & &
\end{tabular}


Con respecto a la prueba $r$ de Pearson para obtener el coeficiente de confiabilidad de la prueba, los resultados mostraron que el coeficiente $r$ de Pearson para el factor poniéndose de pie, el valor obtenido fue de 0.73 , para caminar fue de 0.77 , para subir gradas fue de 0.92 y para el factor de bajar gradas fue de 0.75 , indicando a su vez, que todos los coeficientes $r$ de Pearson fueron mayores que el valor crítico de la Tabla de Pearson, el cual según la Tabla, fue de 0.634 para 11 g.l. y $\alpha$ un de .01 (Ver Tabla 3).

Tabla 3

Coeficiente $r$ Pearson para las cuatro posiciones.

\begin{tabular}{lcc}
\hline & $*$ & $\mathbf{r}$ \\
\cline { 2 - 3 } Poniéndose de pie & 0.634 & 0.73 \\
Caminando & 0.634 & 0.77 \\
Subiendo gradas & 0.634 & 0.92 \\
Bajando gradas & 0.634 & 0.75 \\
\hline
\end{tabular}

* Valor crítico del coeficiente de correlación de Pearson, para 11 g.1., $\alpha=.01$

\section{DISCUSIÓN}

En la segunda parte de los resultados, se observó que todos los valores de la $\mathrm{Fc}$ en los diferentes movimientos evaluados fueron siempre menores a la $\mathrm{Ft}$ (11 g.l., p > .001), por lo tanto se acepta la hipótesis nula propuesta, que indica que se dará una consistencia entre los promedios de la primera medición contra los promedios de la segunda medición para cada uno de los movimientos evaluados.

Este resultado se esperó, puesto que las condiciones entre una y otra medición fueron balanceadas, es decir, al no haber tratamiento entre ambas mediciones, se garantiza que la condición de dolor permanezca constante y por lo tanto no se encontraron diferencias estadísticamente significativas entre los promedios.

Esto último fue vital, pues si al obtener el coeficiente de correlación de Pearson estando las condiciones controladas $\mathrm{y}$, aun así, se hubiera obtenido un coeficiente de correlación bajo y no significativo, entonces se hubiera mostrado que el test no es confiable; caso que no ocurrió; además, aunque solo hubo un coeficiente de correlación alto (subiendo gradas) y aunque en los otros tres (poniéndose de pie, caminando y bajando gradas) los coeficientes de correlación fueron moderados, todos los cuatro coeficientes de correlación de los distintos movimientos medidos estuvieron por encima del valor crítico del coeficiente de correlación de Pearson obtenido y por lo tanto se puede tener la seguridad de que el test es confiable.

\section{Conclusiones}

Con base en los resultados obtenidos, se concluye que el dolor realmente se puede llegar a medir y que además se puede llegar a obtener la confiabilidad

de un test de dolor, siempre y cuando se tengan las condiciones controladas.

El test de dolor realizado, provee pautas para la evaluación del mismo no solo para el área del adulto mayor, sino también para otras áreas en las que se desee implementar una nueva prueba de dolor, pues los resultados sugieren que teniendo un estímulo específico para un problema específico, le va permitir observar el problema del dolor sea a los médicos o a los terapeutas físicos más directamente.

Finalmente, con base en el análisis estadístico del Producto Momento de Pearson y a la Tabla de Significancia de la misma, se establece que el instrumento es realmente confiable para cada una de las cuatro posiciones medidas.

Agradecimiento: Al fisioterapeuta Luis Felipe Medina por su contribución en la parte de marco teórico y revisión y crítica del presente documento.

\section{REFERENCIAS}

Aguilar, R. (1991) En: Ninomiya, J.G. (1991) Fisiología Humana: Neurofisiología. México D.F.: Edit. El Manual Moderno, S. A. de C. V., 146147. 
Ary, D.; Jacobs, L. Ch.; Razavieh, A. (1989). Introducción a la Investigación Pedagógica. $\left(2^{\circ}\right.$ edic.) México D.F.: McGraw - Hill Interamericana de México, S. A. de C. V., p. $214-220$.

Chapman, C. R.; Syrjala, K. L. (1990) En: Bonica, J. J. (1990) The Management of Pain. (2 ed.). Pennsylvania: Lea \& Febiger. Tomo 1, 580-594.

Collins, W.F.; Nulsen, F.E.; Randt, C.T. (1960) Relation of peripheral nerve fibre size and sensation in man. Archives of Neurology 3, 381-385. Cook, D.B., O'Connor, P.J.; Eubanks, S.A.; Smith, J.C., Lee, M. (1997). Naturally ocurring muscle pain during exercise: assessment and experimental evidence. Medicine and Science in Sports and Exercise 29, (8): 999-1012.

Gasser, H.S.; Erlanger, J. (1929) The role of fibre size in the establishment of a nerve block by pressure or cocaine. American Journal of Physiology. $88,581-591$.

Heinbecker, P; Bishop, G.H.; O’Leary, J. (1934) Analysis of sensation in terms of the nerve impulse. Archives of Neurology and Psychiatry. 31, 34-53.

Hernández, R.; Fernández, C.; Baptista, P. (1991). Metodología de la Investigación. México D.F.: McGraw - Hill Interamericana de México, S.A. de C.V., p. 242-248.

Jensen, M.P., Karoly, P. \& Braver, S. (1986). The measurement of clinical pain intensity: a comparison of six methods. Pain, 27, 117-126.

Kerlinger, F. (1988) Investigación del Comportamiento. (2 edic.) México D.F.: McGraw Hill Interamericana de México, S.A. de C.V., p. 459470 .

Lord, F.; Novick, M. (1968) Statistical Theories of Mental Test Scores. Massachussets: Adison-Wesley, 30-31.

O'Connor, P.J.; Cook, D. B. (1999). Exercise and Pain: The Neurobiology, Measurement, and Laboratory Study of Pain in Relation to Exercise in Humans. Exercise and Sport Sciences Reviews, 27: 119-166.

Prieto (1991) En: Ninomiya, J.G. (1991) Fisiología Humana: Neurofisiología. México D.F.: Edit. El Manual Moderno, S. A. de C. V., 166-167168.

Revill, S.I., Robinson, J.O.,Rosen, M. \& Hogg, M.I. (1976). The reliability of a linear analogue for evaluating pain. Anaesthesia 31, 1191-1198.

Schürmann, K. En: Beks, J. (1980) El Manejo del Dolor. México D.F.: Edit. El Manual Moderno, p. 41.

Thomas, J.R.; Nelson, J.K. (1985) Research Methods in Physical Activity. (2da edic.). Illinois: Human Kinetics Publishers, Inc., p. 349-353.

Verbiest, H. En: Beks, J. (1980) EI Manejo

del Dolor. México D.F.: Edit. El Manual Moderno, p. 60-62.

Whiting, W.C., Zernicke, R.F. (1998). Mechanisms of Injury. En Whiting, W.C., Zernicke, R.F. (Eds.),
Biomechanics of Musculoeskeletal Injury (p. 121). Champaign, Illinois, EE.UU: Human Kinetics. Zotterman, Y. (1933) Studies in the peripheral nervous mechanisms of pain. Acta Medica Scandinavica. 80, 185-242. Zwetnow, N. En: Beks, J. (1980) El Manejo del Dolor. México D.F.: Edit. El Manual Moderno, p. 112-113,121-131. 